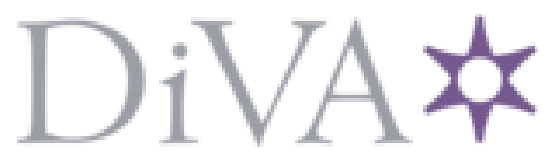

http://www.diva-portal.org

This is the published version of a paper published in Physical Review A. Atomic, Molecular, and Optical Physics.

Citation for the original published paper (version of record):

Jääskeläinen, M. (2012)

Gravitational self-localization for spherical masses.

Physical Review A. Atomic, Molecular, and Optical Physics, 86(5)

http://dx.doi.org/10.1103/PhysRevA.86.052105

Access to the published version may require subscription.

N.B. When citing this work, cite the original published paper.

Permanent link to this version:

http://urn.kb.se/resolve?urn=urn:nbn:se:du-11222 


\title{
Gravitational self-localization for spherical masses
}

\author{
Markku Jääskeläinen \\ Dalarna University, SE-79188 Falun, Sweden \\ (Received 7 August 2012; published 8 November 2012)
}

\begin{abstract}
In this work, I consider the center-of-mass wave function for a homogenous sphere under the influence of the self-interaction due to Newtonian gravity. I solve for the ground state numerically and calculate the average radius as a measure of its size. For small masses, $M \lesssim 10^{-17} \mathrm{~kg}$, the radial size is independent of density, and the ground state extends beyond the extent of the sphere. For masses larger than this, the ground state is contained within the sphere and to a good approximation given by the solution for an effective radial harmonic-oscillator potential. This work thus determines the limits of applicability of the point-mass Newton Schrödinger equations for spherical masses. In addition, I calculate the fringe visibility for matter-wave interferometry and find that in the low-mass case, interferometry can in principle be performed, whereas for the latter case, it becomes impossible. Based on this, I discuss this transition as a possible boundary for the quantum-classical crossover, independent of the usually evoked environmental decoherence. The two regimes meet at sphere sizes $R \approx 10^{-7} \mathrm{~m}$, and the density of the material causes only minor variations in this value.
\end{abstract}

DOI: 10.1103/PhysRevA.86.052105

PACS number(s): 03.65.Ta, 03.65.Ge, 03.75.-b

\section{INTRODUCTION}

In quantum mechanics, the measurement problem remains unsolved and current work on the possible role of gravity in its solution receives increasing attention [1]. Gravitational self-interactions have no natural interpretation using the Born rule [2] and are thus not a part of the orthodox Copenhagen interpretation [3]. It follows that there will be profound implications at the fundamental level if effects due to the gravitational self-interaction can be observed experimentally. It is indisputable that quantum-mechanical objects are sensitive to external gravitational fields, as the effects of this have been observed experimentally with both neutrons [4] and atoms [5]. From a fundamental point of view, it would be desirable to quantize gravity [6,7] for complete consistence, but this still remains a formidable task. Arguments that gravity has to be quantized [8] and experiments claiming support for this [9] are found in the literature. More recently, arguments supporting the possibility of combining classical gravity with quantum mechanics have become more common [10], pointing to the conclusion that the only real reason for requiring a quantization of gravity at this point would be philosophical rather than empirical or logical [11]. The inclusion of self-interaction terms is thus not prohibited, and detecting their presence seems to force a realistic interpretation of the wave function as density of mass in configuration space, which is a very intuitive interpretation. After initially considering this as a possibility [12], Schrödinger rejected that the solutions of the wave equation represent reality, partly due to an exchange of letters with Lorentz [13], where he came to realize some of the difficulties with this interpretation. Those of interest here are the spreading of wave packets and the problem of many-particle wave functions. The latter has a configuration space that is $3 N$ dimensional, thus making it hard to interpret the wave function $\Psi(\vec{r})$ as giving the mass density. A possible solution to these problems is the inclusion of self-gravitational interactions to localize the wave function in space [14]. Here I study the influence of mass and density on the localization properties of the ground state of a self-gravitating sphere. In Sec. II, I introduce the model and derive the stationary-state
Schrödinger equation for a spherical geometry. Section III covers the results from the numerical solution for the ground state and also discusses the calculation of fringe visibility for matter-wave interferometry using these states. Finally, Sec. IV concludes with a discussion of the results and their relation to some previous works and possible implications for proposed experiments.

\section{MODEL}

The main feature of gravity is that it is always attractive and will focus free wave functions into bound states [14,15]. For a nonrelativistic system of $N$ charged particles under the influence of gravitational self-interactions [14], the state is determined by the stationary Schrödinger equation

$$
\begin{aligned}
E \Psi_{N}\left(\vec{r}^{N}\right)= & -\sum_{i} \frac{\hbar^{2}}{2 m_{i}} \nabla_{\vec{r}_{i}}^{2} \Psi_{N}\left(\vec{r}^{N}\right)+V_{\mathrm{EM}}\left(\vec{r}^{N}\right) \Psi_{N}\left(\vec{r}^{N}\right) \\
& +U_{\mathrm{G}}^{\text {Self }}\left[\Psi_{N}\left(\vec{r}^{N}\right)\right] \Psi_{N}\left(\vec{r}^{N}\right),
\end{aligned}
$$

where the Coulomb interaction between the particles is

$$
V_{\mathrm{EM}}\left(\vec{r}^{N}\right)=\sum_{i \neq j} \frac{q_{i} q_{j}}{\left|\vec{r}_{i}-\vec{r}_{j}\right|},
$$

and the Newtonian self-interaction is [14]

$$
U_{\mathrm{G}}^{\text {Self }}\left[\Psi_{N}\left(\vec{r}^{N}\right)\right]=-G \sum_{i} m_{i}^{2} \int \frac{\left|\Psi_{N}\left(\vec{r}^{\prime}\right)\right|^{2}}{\left|\vec{r}_{i}-\vec{r}_{i}^{\prime}\right|} d V^{\prime N},
$$

where $d V^{\prime N}$ denotes the volume element for the source point $\vec{r}^{\prime N}$ in $3 N$-dimensional space. My goal here is mainly to study the center-of-mass wave function for solid materials under nonrelativistic and nonextreme conditions for densities corresponding to solid materials. It is then instructive to transform into center-of-mass

$$
\vec{r}=\sum_{i} m_{i} \vec{r}_{i} / M
$$

and relative coordinates

$$
\vec{\rho}_{i}=\vec{r}_{i}-\vec{r}
$$


where

$$
M=\sum_{i} m_{i}
$$

is the total mass and there are $N-1$ relative coordinate vectors. As gravitation is much weaker than electromagnetism and the interparticle electromagnetic potential only depends on the relative coordinates, the center-of-mass dependence can be separated out by the ansatz

$$
\Psi_{N}\left(\vec{r}, \vec{\rho}^{N-1}\right)=\Psi(\vec{r}) \Phi\left(\vec{\rho}^{N-1}\right),
$$

where the relative coordinate wave function obeys

$$
\begin{aligned}
E_{\Phi} \Phi\left(\vec{\rho}^{N-1}\right)= & -\sum_{i} \frac{\hbar^{2}}{2 m_{i}} \nabla_{\vec{\rho}_{i}}^{2} \Phi\left(\vec{\rho}^{N-1}\right) \\
& +V_{\mathrm{EM}}\left(\vec{\rho}^{N-1}\right) \Phi\left(\vec{\rho}^{N-1}\right) .
\end{aligned}
$$

In principle, there will also be a gravitational interparticle interaction, which is not included here as it will be negligible in comparison with the Coulomb interaction between particles. For the center-of-mass wave function of a charge-neutral system such that $\sum_{i} q_{i}=0$, the electromagnetic interaction does not depend on the center-of-mass coordinate, and we have

$$
\varepsilon \Psi(\vec{r})=-\frac{\hbar^{2}}{2 M} \nabla_{\vec{r}}^{2} \Psi(\vec{r})+\int V_{G}\left(\vec{r}-\vec{r}^{\prime}\right)\left|\Psi\left(\vec{r}^{\prime}\right)\right|^{2} d V^{\prime},
$$

where $\varepsilon=E-E_{\Phi}$, and the effective potential is given by the mass distribution of the relative wave function. We can interpret $V_{G}$ as the gravitational potential at the position $\vec{r}$ due to a mass distribution centered at the position $\vec{r}^{\prime}$ with density given by the relative wave function $\Phi\left(\vec{\rho}^{N-1}\right)$, integrated over the internal degrees of freedom. For the case of a solid sphere, i.e., constant density within a radius $R$, we have, for the potential at a radial distance $r=|\vec{r}|$ from the center,

$$
V_{G}(r)= \begin{cases}-\frac{G M^{2}}{R}\left(\frac{3}{2}-\frac{r^{2}}{2 R^{2}}\right) & \text { if } \quad r \leqslant R \\ -\frac{G M^{2}}{r} & \text { if } r \geqslant R .\end{cases}
$$

Outside of $R$, the potential is identical to that due to a pointlike distribution, whereas inside of $R$, the spherical symmetry leads to shielding of the exterior shell of mass, and only the mass inside of the radial distance $r$ will contribute, causing a parabolic dependence of the potential. This is a consequence of the gravitational Gauss's law for the mass distribution.

\section{RESULTS}

Equation (9) is a nonlinear integro-differential equation where the effective gravitational potential is given by averaging the potential $V_{G}(r)$ over center-of-mass positions weighted by $|\Psi(\vec{r})|^{2}$. As a result, the potential will be smeared out over the region which the center-of-mass wave function is localized over. In principle, both the mass $M$ and the radius $R$ are parameters that can be varied. A solid sphere of radius $R$ and density $\varrho$ has a mass

$$
M=4 \pi R^{3} \varrho / 3 .
$$

For convenience, I choose to vary the mass and the density as parameters. The density of solid elements varies from $\varrho=534 \mathrm{~kg} \mathrm{~m}^{-3}$ for lithium to $\varrho=22610 \mathrm{~kg} \mathrm{~m}^{-3}$ for osmium, which is the densest. The assumption of constant density breaks down at the surface where boundary effects lower the density [16]. For small-enough spheres, around tens of nanometers, this leads to changes in the average density. I note that lower values than that given above for lithium can be achieved by nanostructuring the spheres while still retaining an approximately homogenous density, but do not consider such cases here. For large masses, the wave function becomes increasingly localized, and when it is approximately nonzero only deep within the radius of the mass distribution, it effectively sees a spherical harmonic-oscillator potential with frequency

$$
\omega=\sqrt{M G / R^{3}}=\sqrt{\frac{4 \pi}{3} \varrho G} .
$$

For large masses, the true ground state is thus expected to be reasonably well approximated by the Gaussian ground state of a spherical oscillator with frequency given by Eq. (12).

In the general case, no analytical solution exists to Eqs. (9) and (10). For any given combination of density and total mass, I solve Eq. (9) numerically for a spherical distribution with the potential given by Eq. (10) using a pseudospectral method [17,18] combined with Gauss-Laguerre integration for the interaction [19] to obtain the ground state self-consistently with high accuracy. In Fig. 1, the wave function $\Psi(r)$ and the averaged potential are shown for a mass $M=2 \times 10^{-17} \mathrm{~kg}$. Also shown are the bare potential, given by Eq. (10), and the ground state of a harmonic oscillator corresponding to the minima, where both are shown with dashed lines. We see in Fig. 1 that the averaging produces a significantly changed effective potential in comparison with the bare one given by Eq. (10). The wave function is confined to larger radii as a result of this.

My main objective here is to investigate how the ground state changes as a function of mass and elemental density.

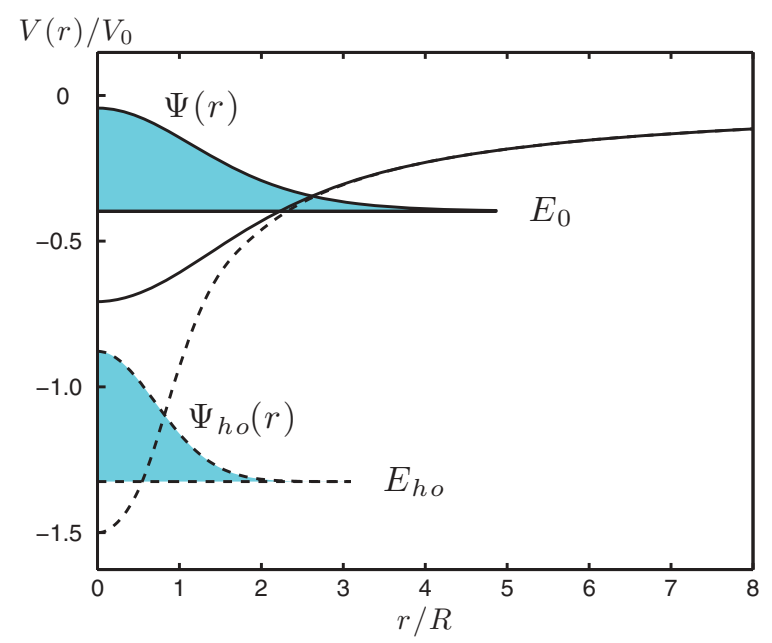

FIG. 1. (Color online) Wave function $\Psi(r)$, shown shaded, and potential for a sphere of osmium with $M=2 \times 10^{-17} \mathrm{~kg}$. The bare potential given by Eq. (10) divided by $V_{0}=G M^{2} / R$, as well as the ground-state wave function for the harmonic-oscillator approximation, are also shown, both with dashed lines. It can be seen that the effective potential is changed by the averaging and becomes less deep, while the ground state becomes less localized. 
Of special interest is the radial extent, measured through the average radius,

$$
\langle r\rangle=4 \pi \int_{0}^{\infty} \Psi(r)^{2} r^{3} d r,
$$

since this will determine most of the stationary properties. For a sufficiently localized wave function, we can calculate the average radius of the ground state in the harmonic approximation of the potential using the oscillator frequency in Eq. (12), and arrive at

$$
\left\langle r^{\mathrm{ho}}\right\rangle=\left(\frac{12 \hbar^{2}}{M^{2} \pi^{3} G \varrho}\right)^{\frac{1}{4}}<R .
$$

The wave function for large values of the total mass thus has the character of a core state embedded within the radius of the sphere. For the opposite case, when the size of the sphere becomes negligible, we can treat it as a point source. In this case, the Schrödinger equation can be recast as $[15,20,21]$

$$
E \Psi(r)=-\frac{\hbar^{2}}{2 M} \frac{1}{r^{2}} \frac{\partial}{\partial r}\left[r^{2} \frac{\partial \Psi(r)}{\partial r}\right]+\Phi_{G}(r) \Psi(r),
$$

where the gravitational potential $\Phi_{G}(r)$ obeys the Poisson equation

$$
\frac{1}{r^{2}} \frac{\partial}{\partial r}\left[r^{2} \frac{\partial \Phi_{G}(r)}{\partial r}\right]=-4 \pi G M^{2} \Psi(r)^{2} .
$$

The equation system (15) and (16) can be solved numerically, and we find, for the average radius,

$$
\left\langle r^{\mathrm{NS}}\right\rangle=c_{\mathrm{NS}} \frac{1}{\hbar^{2} G M^{3}}=c_{\mathrm{NS}} r_{\mathrm{GB}} .
$$

In Eq. (17), the coefficient $c_{\mathrm{NS}} \approx 4.194$ was determined numerically, and the self-gravitational Bohr radius defined by

$$
r_{\mathrm{GB}}=1 / \hbar^{2} G M^{3}
$$

for a mass $M$ is introduced. The radius $r_{\mathrm{GB}}$ corresponds to the expectation value of the radial coordinate for a mass distribution of negligible extent, i.e., a point mass. The natural length scale for the Newton-Schrödinger system is then the self-gravitational Bohr radius when the mass is low enough that the source can be approximated by a distribution with negligible extent. For the general case, I solve Eq. (9) using Eq. (10) and evaluate Eq. (13) numerically for different values of the mass and density. Figure 2 shows the resulting average radius as a function of total mass for the densities of lithium and osmium. In addition, the sizes of solid spheres as well as the resulting size for the harmonic-oscillator approximation, given by Eq. (14), and the value for a point mass, given by Eq. (17), are shown by dashed lines. The choice of osmium and lithium brackets the possible experimental choices of materials and shows what the limits are for ground-state size, regardless of material. We see from Fig. 2 that the average radii for the exact solution approaches the values given by Eq. (14) for large masses, and also approaches those of Eq. (17) for small masses. A crossover between the large and small mass approximations occurs around $M \approx 10^{-17} \mathrm{~kg}$, when the average radius approximately equals that of a solid sphere. This mass range marks a boundary above which the center-of-mass coordinate and its associated wave function cannot be taken

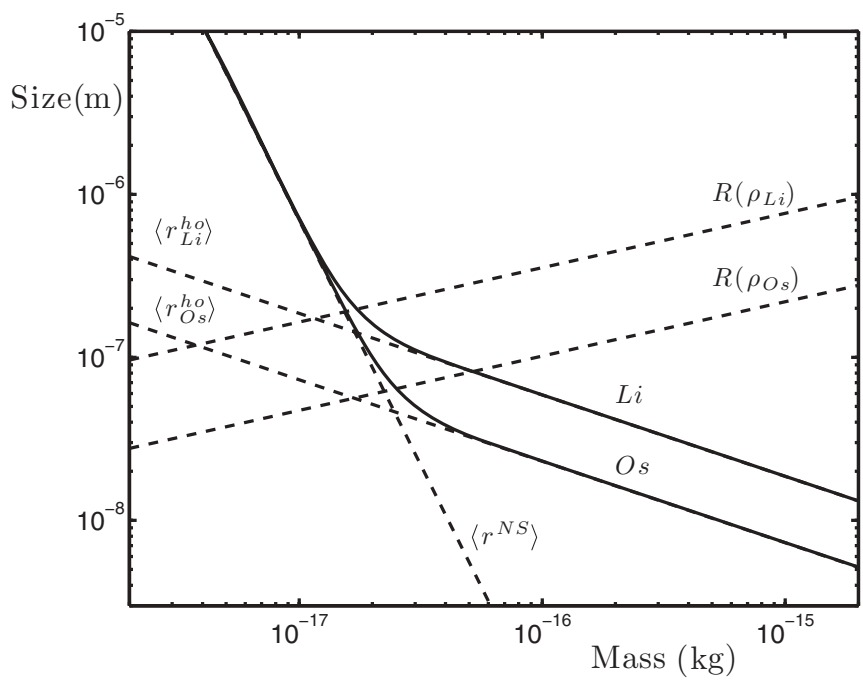

FIG. 2. Average radius vs mass for the least (lithium) and most (osmium) dense solid materials is shown by solid lines. Also, the average radii for the harmonic-oscillator approximation, given by Eq. (14), and for the point source, given by Eq. (17), are shown by dashed lines. Curves are given for both lithium and osmium, as labeled.

as the source for gravity. The system is no longer pointlike, but is instead spatially extended, i.e., the details of the actual mass distribution of the many-body state become important. We also note that the crossover changes only little with the density of the sphere. The crossover also marks the boundary between states that have the center-of-mass wave functions localized within the actual mass distribution of the individual particles. As a consequence of the crossover, the applicability of the point-source model given by Eqs. (15) and (16) is limited to masses below approximately $10^{-17} \mathrm{~kg}$, unless the density is considerably higher than those of solids under normal conditions. For higher masses, the spherical shells outside the wave function give no contribution to the potential, and the wave function effectively experiences only the gravitational field from a fraction of the mass. As a result of this shielding, the average radius depends on the mass more weakly, which is something that can be seen in Fig. 2 for masses above the crossover at approximately $10^{-17} \mathrm{~kg}$. It is important to know what the possible experimental consequences are of this localization of the center-of-mass wave function. Interference of matter waves is a fundamentally quantum-mechanical property not observed for classical massive objects. The presence of interference can thus be taken as a boundary between classical and quantum mechanics, and its absence is often attributed to environmental decoherence [22]. Here I consider the alternative that interference disappears due to localization. In order to investigate the effects due to this, I calculate a measure for the fringe visibility in a double slit or diffraction-grating experiment. The visibility will be limited by the spatial envelope, and can be estimated by the correlator

$$
V=\langle\psi(\vec{r}) \psi(\vec{r}+d \hat{z})\rangle,
$$

where I have assumed a grating or slit with period $d$ to be oriented along $\hat{z}$, and also oriented perpendicularly to the center-of-mass motion. In principle, a detailed dynamical 


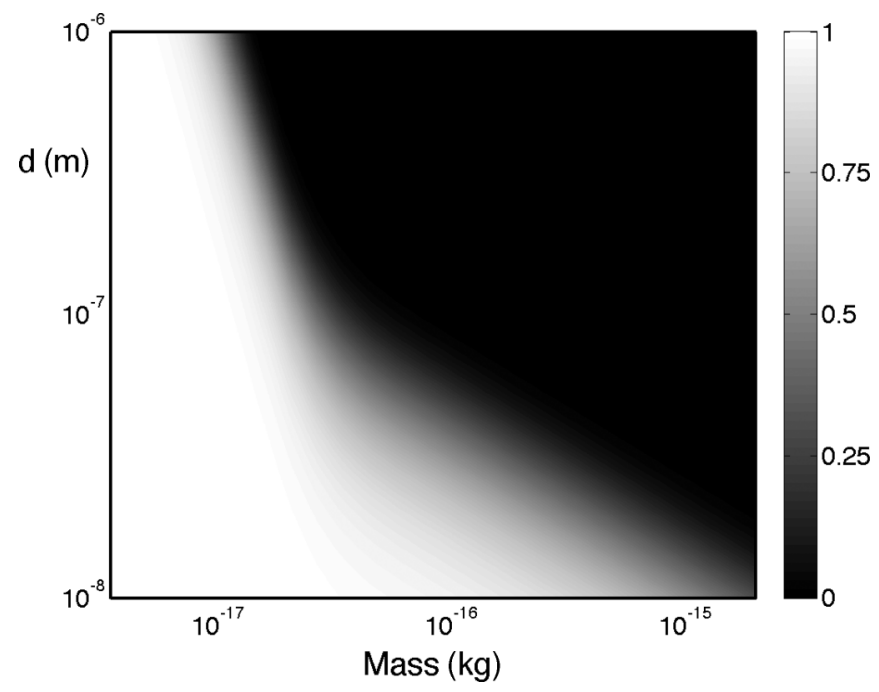

FIG. 3. Visibility as a function of mass and grating period for osmium. The visibility is seen to decrease rapidly for grating periods larger than the average radius.

investigation would be needed to determine the exact value of the visibility in any given experiment to include any effects of the interaction between the grating and the wave function. It is well known that Eq. (19) offers a reasonable estimate based on coherence theory [23]. If the visibility calculated by Eq. (19) is low, then it is very unlikely that interference fringes will actually be observed. The visibility calculated here thus serves as a reliable upper bound on the fringe visibility that one would expect to find in experiments. The visibility is shown in Fig. 3 as a function of mass and diffraction grating $d$ for osmium. The visibility decreases rapidly when $d\rangle\langle r\rangle$, i.e., when the grating period becomes larger than the average radius.

\section{DISCUSSION}

One conclusion is that matter-wave interference experiments using the center-of-mass distribution of particles become exceedingly difficult when the mass is larger than $M \approx 10^{-17} \mathrm{~kg}$. For masses larger than this, the spheres will basically behave as classical particles, as quantum interference effects will be suppressed. Current matter-wave interferometers $[24,25]$ operate well within the nonlocalized regime where the visibility is high, and would need to increase by orders of magnitude in particle mass to start probing the transition to classicality caused by gravity. Instead of observing the center-of-mass distribution of spherical masses, another option is to entangle the center-of-mass dynamics with a light field in a cavity, as suggested in [26,27]. In both of the suggested experiments, the ranges of the trapped center-of-mass wave function are localized by trapping cavity fields resulting in widths around $10^{-10}-10^{-12} \mathrm{~m}$, which is far below the values one finds from solving Eq. (9) for the parameters in question. The wave function is thus dominated by the optical trap with the self-gravity contributing negligibly. The prospects to observe any effects due to the gravitational self-interaction in the trapped states are thus likely to be negligible. It is, however, worth noting that in [26], the masses considered, i.e., around $10^{-20}-10^{-18} \mathrm{~kg}$, are below the crossover, whereas the example for a sphere considered in [27] is $M \approx 10^{-16} \mathrm{~kg}$, on the other side. The center-of-mass wave function of the spheres considered in these two experiments would thus behave very differently if passed through an interferometric setup as free wave packets. From the results here, it appears that the best possibility to observe the crossover directly would be to use an interferometer with a light grating formed as a standing wave in the infrared range $\lambda \approx 10^{-6} \mathrm{~m}$, sending spheres of a dense material through it and recording the far-field distribution of center-of-mass positions. Varying the mass and determining the dependence of the fringe visibility on it would thus serve as an indicator of the transition.

In [28], the authors consider the dynamics of the NewtonSchrödinger system for a pointlike mass when the initial state is a Gaussian of initial width, for concreteness taken to be half a micron, which in principle could be significantly different from the ground-state size. We note here that the results in [28] for masses above $M \approx 2 \times 10^{-17} \mathrm{~kg}$ are not valid in the sense that the point-mass model is not applicable. This is because there is no naturally occurring element with sufficient density to produce a sphere of negligible extent. Above this value, it is necessary to take the spatial extent of the source into account, and the dynamics will be different.

The physical picture investigated here is that the wave function in $3 N$ dimensions separates into $3(N-1)$ relative coordinates that give rise to a bound state, which corresponds to a mass distribution. This mass distribution in turn acts as a source for the self-gravitational field for the center-of-mass degree of freedom. The question remains, however, whether the approach used here to combine nonrelativistic quantum mechanics with Newtonian gravity is a physically acceptable one. It is instructive to compare with the situation in electrodynamics, where field quantization has been achieved and semiclassical approximations for field amplitudes are known to accurately describe the physics as long as the fluctuations in the amplitude do not influence the dynamics. For gravity, large curvatures correspond to large fluctuations in the metric, which would then in a naive manner correspond to large fluctuations in the gravitational field around its expectation value. Here we expect that the assumption that $\langle r\rangle \gg l_{P}$ holds, which implies that the ground state is smooth over distances that are large compared with the Planck length. This is always true since the mass needed to reach the Planck length will create a classical black hole. From this, it is apparent that the assumption of Newtonian gravity must break down in such cases, and our approach will clearly be invalidated. Already before this, for smaller masses, the validity of the continuum approximation implicit in this work is expected to be violated when $\langle r\rangle \approx 10^{-10} \mathrm{~m}$ is reached, since this corresponds to typical values of lattice parameters for solids.

The inclusion of self-interaction terms in Schrödinger equations lacks an obvious interpretation within the Copenhagen school of thought [3]. The detection of their presence then suggests a realistic interpretation of the wave function as physical density. In addition, the inclusion of self-gravitation in the nonrelativistic Schrödinger equation has the attractive feature that a $3 N$-dimensional wave function will separate into relative and center-of-mass coordinates, and taken together with the localization of bound states for both suggests that the wave function can correspond to real objects. For mass 
values above the crossover, the center-of-mass wave function is confined within the mass distribution of the individual particles. The composite system then behaves as a classical sphere with a trajectory that is well described by Ehrenfest's theorem, and interacts with other similar objects through collisional events in a mostly classical way.

To conclude, I performed an accurate, numerical solution of the Schrödinger equation for a spherical mass distribution with the inclusion of the self-gravitational interaction, and quantified the crossover between weakly localized ground states and ground states localized within the mass distribution. I found that the mass and radius where the crossover occurs are $M \approx 10^{-17} \mathrm{~kg}$ and $R \approx 10^{-7} \mathrm{~m}$, respectively, which are values found to be only weakly dependent of the density. The visibility for matter-wave interferometry exhibits the same type of crossover, thus verifying a boundary between classical and quantum behavior for the center-of-mass coordinate. The results here apply only to spherical masses, but should serve as a starting point for further investigations to determine under what conditions solids of different shapes will exhibit localization. Effects due to different geometries will affect the exact location of the crossover, which is something likely to be of importance to experimental searches for gravitational effects in quantum objects [29].
[1] A. Bassi, K. Lochan, S. Satin, T. P. Singh, and H. Ulbricht, arXiv:1204.4325.

[2] M. Born, Z. Phys. 37, 863 (1926); 38, 803 (1926).

[3] S. L. Adler, J. Phys. A 40, 755 (2007).

[4] V. V. Nezvizhevsky et al., Nature (London) 415, 297 (2002).

[5] C. G. Aminoff, A. M. Steane, P. Bouyer, P. Desbiolles, J. Dalibard, and C. Cohen-Tannoudji, Phys. Rev. Lett. 71, 3083 (1993).

[6] C. Kiefer, Quantum Gravity (Oxford University Press, Oxford, UK, 2007).

[7] C. Rovelli, Quantum Gravity (Cambridge University Press, Cambridge, UK, 2004).

[8] K. Eppley and E. Hannah, Found. Phys. 7, 51 (1977).

[9] D. N. Page and C. D. Geilker, Phys. Rev. Lett. 47, 979 (1981).

[10] M. Albers, C. Kiefer, and M. Reginatto, Phys. Rev. D 78, 064051 (2008).

[11] J. Mattingly, Phys. Rev. D 73, 064025 (2006).

[12] E. Schrödinger, Annalen der Physik 79, 361 (1926); 79, 489 (1926); 80, 437 (1926); 81, 109 (1926).

[13] Letters on Wave Mechanics, edited by K. Przibram (Philosophical Library, New York, 1967).

[14] L. Diosi, Phys. Lett. A 105, 199 (1984).

[15] R. Ruffini and S. Bonazzola, Phys. Rev. 187, 1767 (1969).

[16] K. T. Ramesh, Nanomaterials: Mechanics and Mechanisms (Springer, New York, 2009).

[17] B. Fornberg, A Practical Guide to Pseudospectral Methods (Cambridge University Press, Cambridge, UK, 1996).
[18] J. A. C. Weideman and S. C. Reddy, ACM Trans. Math. Software 26, 465 (2000).

[19] P. K. Kythe and M. R. Schäferkotter, Handbook of Computational Methods for Integration (Chapman and Hall/CRC, Boca Raton, 2004).

[20] R. Penrose, Phil. Trans. R. Soc. A 356, 1927 (1998).

[21] I. Moroz, R. Penrose, and P. Tod, Class. Quant. Grav. 15, 2733 (1998).

[22] E. Joos et al., Decoherence and the Appearance of a Classical World in Quantum Theory (Springer, Heidelberg, 2003).

[23] L. Mandel and E. Wolf, Optical Coherence and Quantum Optics (Cambridge University Press, Cambridge, UK, 1995).

[24] A. Cronin, J. Schmiedmaayer, and D. Pritchard, Rev. Mod. Phys. 81, 1051 (2009).

[25] M. Arndt and K. Hornberger, in Quantum Coherence in Solid State Systems, edited by B. Deveaud-Pledran, A. Quattropani, and P. Schwendimann, International School of Physics "Enrico Fermi," Course CLXXI, Vol. 171 (IOS Press, Amsterdam, 2009).

[26] D. E. Chang et al., Proc. Natl. Acad. Sci. 107, 1005 (2010).

[27] O. Romero-Isart, M. L. Juan, R. Quidant, and J. I. Cirac, New J. Phys. 12, 033015 (2010).

[28] D. Giulini and A. Großardt, Class. Quant. Grav. 28, 195026 (2011).

[29] W. Marshall, C. Simon, R. Penrose, and D. Bouwmeester, Phys. Rev. Lett. 91, 130401 (2003). 